\title{
Extraction and physicochemical properties of low free fatty acid crude palm oil.
}

\begin{abstract}
Response surface methodology was applied to optimize the pre-treatment of oil palm (Elaeis guineensis) fruit spikelets before oil extraction. The treatment applied was drying at different times and temperatures. The dried spikelets were then subjected to mechanical processes and crude oil was extracted. A central composite design was employed to study the responses, namely percentage of free fatty acids (FFA) and oil yield, and the optimum conditions for minimum FFA and maximum oil yield were identified from their respective contour plots. It was concluded that the pre-treatment should be carried out for $12.8 \mathrm{~h}$ at $66.8^{\circ} \mathrm{C}$. Under the optimum conditions, the corresponding response values for FFA and oil yield were $1 \%$ and $33.6 \%$, respectively. Some of the physicochemical properties of the extracted oil were then determined. The low free fatty acid crude palm oil exhibited good physicochemical properties and could be useful for industrial applications.
\end{abstract}

Keyword: RSM; Low free fatty acid crude palm oil; Optimum pre-extraction conditions; Physicochemical properties. 